\title{
Automates et valeurs de transcendance du logarithme de Carlitz
}

\author{
par
}

VALÉRIE Berthé (Marseille)

1. Introduction. Soit $\mathbf{F}_{q}$ le corps de cardinal $q$. Soit $p$ la caractéristique de $\mathbf{F}_{q}$. On définit par analogie avec le cas réel :

$$
\begin{gathered}
\mathbf{Z}=\mathbf{F}_{q}[x], \quad \mathbf{Q}=\mathbf{F}_{q}(x), \\
\mathbf{R}=\mathbf{F}_{q}((1 / x))=\left\{\sum_{n=-\infty}^{\infty} \frac{a_{n}}{x^{n}}: a_{n} \in \mathbf{F}_{q} \text { et les }\left(a_{n}\right)_{n<0} \text { presque tous nuls }\right\} .
\end{gathered}
$$

Le corps $\mathbf{R}$ est le complété de $\mathbf{Q}$ pour la valuation $1 / x$-adique. Enfin, on définit $\mathbf{C}$ comme le complété d'une clôture algébrique de $\mathbf{R} ; \mathbf{C}$ est donc algébriquement clos.

Carlitz a défini dans [4] deux fonctions $\psi$ et $\lambda$ sur $\mathbf{F}_{q}((1 / x))$, qui jouent respectivement les rôles de l'exponentielle et du logarithme réels. Ces fonctions sont ainsi définies :

$$
\begin{gathered}
\psi(t)=\sum_{k=0}^{\infty} \frac{(-1)^{k} t^{q^{k}}}{F_{k}} \quad \text { pour tout } t \text { de } \mathbf{F}_{q}((1 / x)), \\
\lambda(t)=\sum_{k=0}^{\infty} \frac{t^{q^{k}}}{L_{k}} \quad \text { pour tout } t \text { tel que } d^{\circ} t \leq 1,
\end{gathered}
$$

avec

$$
[k]=x^{q^{k}}-x, \quad F_{k}=[k][k-1]^{q} \ldots[1]^{q^{k-1}}, \quad L_{k}=[k][k-1] \ldots[1] .
$$

Carlitz a montré que l'on peut étendre la définition de $\lambda$ à $\mathbf{F}_{q}((1 / x))$ (voir [4]). La fonction $\lambda$ ainsi obtenue est alors l'inverse de la fonction $\psi$. Carlitz a également montré l'existence d'une période pour la fonction $\psi$ :

$$
\forall t \in \mathbf{R}, \forall E \in \mathbf{Z}, \quad \psi(t+E \xi)=\psi(t),
$$

avec

$$
\xi=\left(x^{q}-x\right)^{1 /(q-1)} \Pi \quad \text { et } \quad \Pi=\prod_{j=0}^{\infty}\left(1-\frac{x^{q^{j}}-x}{x^{q^{j+1}}-x}\right) .
$$


La série formelle $\Pi$ joue bien évidemment le rôle du réel $\pi$ et $\xi$ est l'analogue de $2 i \pi$.

Wade a montré diverses propriétés de transcendance concernant ces deux fonctions, qui mettent en évidence l'analogie avec le cas réel. Il a établi, en particulier, deux résultats à mettre en correspondance avec les théorèmes de Hermite-Lindemann et de Gelfond-Schneider, à savoir :

- Si $\alpha$ est un élément non nul de $\mathbf{F}_{q}((1 / x))$ algébrique sur $\mathbf{F}_{q}(x)$, alors $\psi(\alpha)$ et $\lambda(\alpha)$ sont transcendants (voir [19]).

- Si $\alpha$ est non nul et $\beta$ un élément de $\mathbf{F}_{q}((1 / x))$ irrationnel alors l'un des trois nombres $\alpha, \beta, \psi(\beta \lambda(\alpha))$ est transcendant. Si $\alpha$ est nul et si l'on remplace $\lambda(0)$ par $E \xi$, où $E$ appartient à $\mathbf{F}_{q}[x]$ et $\xi=\left(x^{q}-x\right)^{1 /(q-1)} \Pi$, alors la conclusion reste vraie (voir [20]).

On déduit, en particulier, du premier de ces résultats, la transcendance de $\Pi$. Yu a également montré, dans [23] et [24], ces deux propriétés, dans un cadre plus général.

Carlitz a également défini sur $\mathbf{F}_{q}(x)$ une fonction $\zeta$, analogue à la fonction $\zeta$ de Riemann. Elle est définie de la façon suivante :

$$
\zeta(m)=\sum_{G \in \mathbf{F}_{q}[x] \text { et } G \text { unitaire }} 1 / G^{m}, \quad m \geq 1 .
$$

Il existe plusieurs méthodes conduisant à des résultats de transcendance sur les valeurs des fonctions $\psi, \lambda$ et $\zeta$ de Carlitz (voir [21]) :

- la méthode de Wade reprise par Dammame et Hellegouarch, ainsi que par Thakur; elle est à certains égards l'analogue de la méthode classique pour les nombres réels (voir [9]-[12], [17] et [18]),

- les modules de Drinfeld utilisés par Yu; il s'agit d'une généralisation des courbes elliptiques (voir [14] et [15]); c'est la méthode la moins élémentaire mais aussi celle qui donne actuellement le plus de résultats (voir [22]),

- les mesures d'irrationalité sur lesquelles travaillent de Mathan et Chérif (voir [5]-[7]),

- enfin les automates qui ont permis à Allouche de donner une preuve "élémentaire" de la transcendance de la période $\Pi$ de l'exponentielle.

Il s'agit ici de généraliser cette dernière méthode pour l'étendre à d'autres résultats.

En fait, Allouche a montré dans [1], en utilisant les automates et plus précisément le théorème de Christol, Kamae, Mendès France et Rauzy, la transcendance de $\alpha / \Pi$, avec

$$
\alpha=\prod_{j=0}^{\infty}\left(1-\frac{x^{q^{j}}}{x^{q^{j+1}}}\right) .
$$


En élevant $\alpha$ à la puissance $q$, on constate que $\alpha$ est algébrique sur $\mathbf{F}_{q}(x)$. On déduit donc la transcendance de $\Pi$ de celle de $\alpha / \Pi$.

Rappelons l'énoncé du théorème de Christol, Kamae, Mendès France et Rauzy (voir [8]) :

ThÉorème 1 (Christol, Kamae, Mendès France et Rauzy). Soit $(u(n))_{n \in \mathbb{N}}$ une suite à valeurs dans $\mathbf{F}_{q}$. Il y a équivalence entre les deux conditions suivantes:

1. La série formelle $\sum_{n \geq 0} u(n) x^{-n}$ est algébrique sur $\mathbf{F}_{q}(x)$.

2. L'ensemble $E$ des sous-suites de la suite $(u(n))_{n \in \mathbb{N}}$ défini par

$$
E=\left\{\left(u\left(q^{k} n+r\right)\right)_{n \in \mathbb{N}}: k \geq 0,0 \leq r \leq q^{k}-1\right\}
$$

est fini.

Nous nous proposons de donner ici une preuve "automatique" du résultat suivant :

ThÉORÈme 2. Soit $P$ une fraction de la forme $P=\sum_{v>-1} p_{v}(1 / x)^{v}$ où $p_{v} \in \mathbf{F}_{q}$, et $p_{v}=0$ pour $v$ assez grand. Alors $\lambda(P) / \Pi^{s}$ est transcendant sur $\mathbf{F}_{q}(x)$, pour $1 \leq s \leq q-3$ et pour $q \neq 2, q \neq 3$.

Nous étudierons en fait le quotient $\frac{\alpha^{s}}{\Pi^{s}} \lambda(P)$. Multiplier par $\alpha^{s}$ permet d'obtenir un développement simple en série formelle de $\frac{\alpha^{s}}{\Pi^{s}} \lambda(P)$ (afin d'utiliser le théorème de Christol, Kamae, Mendès France et Rauzy) sans pour autant influer sur la transcendance de $\lambda(P) / \Pi^{s}$, puisque $\alpha$ est algébrique.

Remarques. Pour $q=3$, il est possible de montrer par les automates la transcendance de $\lambda(P) / \Pi$, mais ce résultat demande plus de travail.

On peut également retrouver partiellement et de façon élémentaire, par cette même méthode, un résultat de $\mathrm{Yu}$, à savoir la transcendance de $\zeta(s) / \Pi^{s}$ pour $1 \leq s \leq q-2$ (voir [2] et [3]). Cette propriété de transcendance a été démontrée par Yu dans [22], pour tout $s$ non divisible par $q-1$ (voir aussi [17] et [18]).

Enfin, Y. Hellegouarch a généralisé l'exponentielle de Carlitz en définissant une exponentielle associée à une suite périodique d'endomorphismes. F. Recher a montré, dans [16], par les automates, la transcendance de la période de cette exponentielle généralisée, pour certains choix d'endomorphismes.

2. Quelques développements en série formelle. On a $\lambda(t)=$ $\sum_{k=0}^{\infty} t^{q^{k}} / L_{k}$, pour $t$ tel que $d^{\circ} t \leq 1$. Soit

$$
P=\sum_{v \geq-1} p_{v}(1 / x)^{v}, \quad \text { où } p_{v} \in \mathbf{F}_{q},
$$


et $p_{v}=0$ pour $v$ assez grand. On se restreint à des exposants $v \geq-1$, pour des raisons de convergence.

La fonction $\lambda$ est linéaire sur $\mathbf{F}_{q}$ et $p_{v}=0$, pour $v$ assez grand. On en déduit que

$$
\lambda(P)=\lambda\left(\sum_{v \geq-1} p_{v}(1 / x)^{v}\right)=\sum_{v \geq-1} p_{v} \lambda\left((1 / x)^{v}\right) .
$$

On a donc

$$
\frac{\alpha^{s}}{\Pi^{s}} \lambda(P)=\sum_{v \geq-1} p_{v}\left(\frac{\alpha^{s}}{\Pi^{s}} \lambda\left((1 / x)^{v}\right)\right) .
$$

2.1. Notations. Nous allons introduire dans cette section quelques suites auxiliaires utiles pour le développement en série formelle de $\frac{\alpha^{s}}{\Pi^{s}} \lambda(P)$. Nous allons développer successivement les termes $\frac{\alpha}{\Pi} \lambda\left((1 / x)^{v}\right), \frac{\alpha^{s}}{\Pi^{s}}\left(\lambda\left((1 / x)^{v}\right)\right)$ et enfin $\frac{\alpha^{s}}{\Pi^{s}} \lambda(P)$.

Considérons le terme

$$
\lambda\left((1 / x)^{v}\right)=\sum_{k=0}^{\infty} \frac{(1 / x)^{v q^{k}}}{L_{k}} .
$$

Rappelons que $L_{k}=\prod_{j=1}^{k}\left(x^{q^{j}}-x\right)$. Par conséquent,

$$
\lambda\left((1 / x)^{v}\right)=(1 / x)^{v}+\sum_{k \geq 1}(1 / x)^{v q^{k}} \prod_{j=1}^{k}\left(\frac{1}{x^{q^{j}}-x}\right) .
$$

On a

$$
\alpha=\prod_{j=0}^{\infty}\left(1-\frac{x^{q^{j}}}{x^{q^{j+1}}}\right) \quad \text { et } \quad \Pi=\prod_{j=0}^{\infty}\left(1-\frac{x^{q^{j}}-x}{x^{q^{j+1}}-x}\right) .
$$

On vérifie alors que

$$
\frac{\alpha}{\Pi}=\prod_{j=1}^{\infty}\left(1-(1 / x)^{q^{j}-1}\right)
$$

Il en résulte que

$$
\lambda\left((1 / x)^{v}\right)=(1 / x)^{v}+\sum_{k \geq 1}(1 / x)^{q+\ldots+q^{k-1}+(v+1) q^{k}} \prod_{j=1}^{k} \frac{1}{\left(1-(1 / x)^{q^{j}-1}\right)} .
$$

En multipliant $\lambda\left((1 / x)^{v}\right)$ par $\alpha / \Pi$, on obtient donc

$$
\frac{\alpha}{\Pi} \lambda\left((1 / x)^{v}\right)=\frac{\alpha}{\Pi}(1 / x)^{v}+\sum_{k \geq 1}(1 / x)^{q+\ldots+q^{k-1}+(v+1) q^{k}} \prod_{j=k+1}^{\infty}\left(1-(1 / x)^{q^{j}-1}\right) .
$$


On définit alors les suites $a^{v}=\left(a^{v}(n)\right)_{n \in \mathbb{N}}, b^{v}=\left(b^{v}(n)\right)_{n \in \mathbb{N}}$ et $c^{v}=$ $\left(c^{v}(n)\right)_{n \in \mathbb{N}}$ de la manière suivante :

$$
\begin{gathered}
\frac{\alpha}{\Pi}(1 / x)^{v}=\sum_{n \geq 0} a^{v}(n) x^{-n}, \\
\frac{\alpha}{\Pi}\left(\lambda\left((1 / x)^{v}\right)-(1 / x)^{v}\right)=\sum_{n \geq 0} b^{v}(n) x^{-n}, \\
\frac{\alpha}{\Pi} \lambda\left((1 / x)^{v}\right)=\sum_{n \geq 0} c^{v}(n) x^{-n} .
\end{gathered}
$$

On a donc $c^{v}=a^{v}+b^{v}$.

Considérons maintenant le développement en série formelle de $\frac{\alpha^{s}}{\Pi^{s}} \lambda\left((1 / x)^{v}\right)$. On a

$$
\frac{\alpha^{s}}{\Pi^{s}} \lambda\left((1 / x)^{v}\right)=\frac{\alpha^{s-1}}{\Pi^{s-1}}\left(\frac{\alpha}{\Pi} \lambda\left((1 / x)^{v}\right)\right) .
$$

Soit $A^{s-1}=\left(A^{s-1}(n)\right)_{n \in \mathbb{N}}$ la suite définie par

$$
\frac{\alpha^{s-1}}{\Pi^{s-1}}=\sum_{n \geq 0} A^{s-1}(n) x^{-n}
$$

On a

$$
\frac{\alpha}{\Pi} \lambda\left((1 / x)^{v}\right)=\sum_{n \geq 0} c^{v}(n) x^{-n}
$$

Par conséquent,

$$
\frac{\alpha^{s-1}}{\Pi^{s-1}}\left(\frac{\alpha}{\Pi} \lambda\left((1 / x)^{v}\right)\right)=\left(\sum_{n \geq 0} A^{s-1}(n) x^{-n}\right)\left(\sum_{n \geq 0} c^{v}(n) x^{-n}\right),
$$

c'est-à-dire,

$$
\frac{\alpha^{s}}{\Pi^{s}} \lambda\left((1 / x)^{v}\right)=\sum_{n \geq 0} x^{-n}\left(\sum_{k=0}^{n} A^{s-1}(k) c^{v}(n-k)\right) .
$$

On définit alors les suites $e^{v}=\left(e^{v}(n)\right)_{n \in \mathbb{N}}, f^{v}=\left(f^{v}(n)\right)_{n \in \mathbb{N}}$ et $g_{v}=$ $\left(g^{v}(n)\right)_{n \in \mathbb{N}}$ de la manière suivante :

$$
\begin{aligned}
e^{v}(n) & =\sum_{k=0}^{n} A^{s-1}(k) c^{v}(n-k), \\
f^{v}(n) & =\sum_{k=0}^{n} A^{s-1}(k) a^{v}(n-k), \\
g^{v}(n) & =\sum_{k=0}^{n} A^{s-1}(k) b^{v}(n-k) .
\end{aligned}
$$


On a

$$
e^{v}=f^{v}+g^{v} \quad \text { et } \quad \frac{\alpha^{s}}{\Pi^{s}} \lambda\left((1 / x)^{v}\right)=\sum_{n \geq 0} e^{v}(n) x^{-n} .
$$

On a, enfin,

$$
\frac{\alpha^{s}}{\Pi^{s}} \lambda(P)=\sum_{v \geq-1} p_{v}\left(\frac{\alpha^{s}}{\Pi^{s}} \lambda\left((1 / x)^{v}\right)\right) .
$$

On définit donc, de même, les suites $E=(E(n))_{n \in \mathbb{N}}, F=(F(n))_{n \in \mathbb{N}}$ et $G=(G(n))_{n \in \mathbb{N}}$ :

$$
\begin{aligned}
& E(n)=\sum_{v \geq-1} p_{v} e^{v}(n), \\
& F(n)=\sum_{v \geq-1} p_{v} f^{v}(n), \\
& G(n)=\sum_{v \geq-1} p_{v} g^{v}(n) .
\end{aligned}
$$

On a

$$
\frac{\alpha^{s}}{\Pi^{s}} \lambda(P)=\sum_{n \geq 0} E(n) x^{-n} \quad \text { et } \quad E=F+G .
$$

2.2. Quelques propriétés. Nous allons établir, dans cette section, des propriétés concernant les suites $a^{v}, b^{v}$ (proposition 1), la suite $A^{s-1}$ (proposition 2) et enfin les suites $f^{v}$ et $g^{v}$ (proposition 3 ). Nous aurons besoin du lemme suivant :

Lemme 1. Soit $j \geq 1$. Si $n$ s'écrit sous la forme

$$
n=r+\sum_{l=j+1}^{\infty} \mu_{l}\left(q^{l}-1\right)
$$

avec $\mu_{l} \in\{0,1, \ldots, q-1\}, \mu_{l}=0$ pour l assez grand et $0 \leq r \leq(q-1)\left(q^{j}-1\right)$, une telle décomposition est unique.

Preuve. Considérons une décomposition de $n$ sous la forme (1).

Si $n=r$, on a nécessairement $\mu_{l}=0$ pour tout $l$. Supposons alors $n \neq r$. Soit $L$ le plus grand indice $l$ tel que $\mu_{l} \neq 0$. On a

$$
\forall m \geq 1 \quad(q-1) \sum_{i=1}^{m}\left(q^{i}-1\right)<q^{m+1}-1 .
$$

D'où

$$
r+\sum_{l=j+1}^{L} \mu_{l}\left(q^{l}-1\right)<q^{L+1}-1
$$


Par conséquent, si $n$ admet deux telles décompositions, les indices des plus grands termes non nuls seront égaux. Supposons alors qu'il existe $\left(\delta_{t}\right)_{j+1 \leq t \leq L}$ à coefficients dans $\{0,1, \ldots, q-1\}$ et $\varrho$ avec $0 \leq \varrho \leq$ $(q-1)\left(q^{j}-1\right)$, tels que

$$
n=r+\sum_{l=j+1}^{L} \mu_{l}\left(q^{l}-1\right)=\varrho+\sum_{t=j+1}^{L} \delta_{t}\left(q^{t}-1\right), \quad \text { avec } \mu_{L} \neq 0 \text { et } \delta_{L} \neq 0 .
$$

Supposons, de plus, que $\delta_{L} \neq \mu_{L}$ et que, par exemple, $\mu_{L}>\delta_{L}$. On a alors

$$
n-\delta_{L}\left(q^{L}-1\right) \geq q^{L}-1>\sum_{t=j+1}^{L-1} \delta_{t}\left(q^{t}-1\right)+\varrho=n-\delta_{L}\left(q^{L}-1\right),
$$

ce qui est impossible. Par conséquent, $\delta_{L}=\mu_{L}$. On montre ainsi, par récurrence, que $\mu_{l}=\delta_{l}$ pour $l \geq j+1$. On en déduit alors que $r=\varrho$, ce qui achève la preuve du lemme 1 .

On a les propriétés suivantes concernant les suites $a^{v}, b^{v}, c^{v}$ :

Proposition 1. 1. On a $a^{v}(n) \neq 0$ si et seulement si $n$ s'écrit sous la forme

$v+\sum_{j \geq 1} \varepsilon_{j}\left(q^{j}-1\right), \quad$ avec $\varepsilon_{j}=0$ ou 1 , et $\varepsilon_{j}=0$ pour $k$ assez grand.

2. Si $b^{v}(n) \neq 0$ alors $n$ s'écrit sous la forme

$$
n=q+\ldots+q^{i-1}+q^{i}(v+1)+\sum_{j=i+1}^{\infty} \varepsilon_{j}\left(q^{j}-1\right),
$$

avec $i \geq 1, \varepsilon_{j}=0$ ou 1 , et $\varepsilon_{j}=0$ pour $j$ assez grand.

Nous aurons besoin du lemme suivant dans la preuve de la proposition 1 :

Lemme 2. Soit $\left(a_{k}(n)\right)_{n \in \mathbb{N}}$ la suite définie par

$$
\prod_{j=k+1}^{\infty}\left(1-(1 / x)^{q^{j}-1}\right)=\sum_{n \geq 0} a_{k}(n) x^{-n} .
$$

Si n s'écrit

(3) $n=\sum_{j=k+1}^{\infty} \varepsilon_{j}\left(q^{j}-1\right) \quad$ avec $\varepsilon_{j}=0$ ou 1 , et $\varepsilon_{j}=0$ pour $k$ assez grand, alors $a_{k}(n)=(-1)^{\Sigma_{j=k+1}^{\infty} \varepsilon_{j}}$, sinon $a_{k}(n)=0$.

La preuve de ce lemme résulte immédiatement de l'unicité de la décomposition de $n$ sous la forme (3) qui découle elle-même du lemme 1. 
Preuve de la proposition 1. Rappelons que

$$
\frac{\alpha}{\Pi}=\prod_{j=1}^{\infty}\left(1-(1 / x)^{q^{j}-1}\right)=\sum_{n \geq 0} a_{0}(n) x^{-n} .
$$

Par conséquent,

$$
\frac{\alpha}{\Pi}(1 / x)^{v}=\sum_{n \geq 0} a_{0}(n) x^{-(n+v)}=\sum_{n \geq v} a_{0}(n-v) x^{-n} .
$$

Or $a_{0}(n) \neq 0$ si et seulement si $n$ s'écrit sous la forme (3) selon le lemme 2 , ce qui achève la preuve de 1 .

On a $\left(^{1}\right)$

$\frac{\alpha}{\Pi}\left(\lambda\left((1 / x)^{v}\right)-(1 / x)^{v}\right)=\sum_{n \geq 0} x^{-n} \sum_{k \geq 1} a_{k}\left(n-\left(q+\ldots+q^{k-1}+(v+1) q^{k}\right)\right)$.

Par conséquent, 2 résulte du lemme 2.

R e m a r que. On ne peut rien dire quant à une réciproque dans 2. En effet, il peut exister plusieurs décompositions de $n$ sous la forme (2). Chacune de ces décompositions apporte un coefficient de valeur absolue égale à 1 . Or on est en caractéristique $p$. Par conséquent, on peut éventuellement avoir $b^{v}(n) \equiv 0(p)$, si $n$ s'écrit sous la forme (2). Néanmoins, si la décomposition de $n$ est unique, alors $b^{v}(n) \neq 0$.

Considérons le développement en série formelle de $\alpha^{s-1} / \Pi^{s-1}$. On a la proposition suivante :

Proposition 2. Soit $1 \leq t \leq q-1$. Soit $\left(A^{t}(n)\right)_{n \in \mathbb{N}}$ la suite définie par

$$
\frac{\alpha^{t}}{\Pi^{t}}=\sum_{n \geq 0} A^{t}(n) x^{-n} \text {. }
$$

Sin s'écrit

$$
n=\sum_{j=1}^{\infty} \mu_{j}\left(q^{j}-1\right) \quad \text { avec } \mu_{j} \in\{0,1, \ldots, t\}, \mu_{j}=0 \text { pour } j \text { assez grand },
$$

alors

$$
A^{t}(n)=(-1)^{\Sigma_{j=1}^{\infty} \mu_{j}} \prod_{j=1}^{\infty} \overline{\left(\begin{array}{c}
\mu_{j} \\
t
\end{array}\right)},
$$

$\operatorname{sinon} A^{t}(n)=0$.

$\left(^{1}\right)$ On pose, pour $n<0, a_{k}(n)=0$. 
Preuve. On a

$$
\begin{aligned}
& \prod_{j=1}^{\infty}\left(1-(1 / x)^{q^{j}-1}\right)^{t}=\left(\sum_{n \geq 0} a_{0}(n) x^{-n}\right)^{t} \\
&=\sum_{n \geq 0} x^{-n}\left(\sum_{n_{i} \geq 0} \text { et } \Sigma_{i=1}^{t} n_{i}=n\right. \\
&\left.\prod_{i=1}^{t} a_{0}\left(n_{i}\right)\right) .
\end{aligned}
$$

Soit $n \in \mathbb{N}$. Soient $n_{1}, \ldots, n_{t}$ tels que $n=\sum_{i=1}^{t} n_{i}, n_{i} \geq 0$ pour tout $i$ et $\prod_{i=1}^{t} a_{0}\left(n_{i}\right) \neq 0$. D'après le lemme 2 , il existe $\left(\varepsilon_{i, j}\right)$ avec $\varepsilon_{i, j}=0$ ou 1 , et $\varepsilon_{i, j}=0$ pour tout $i$ et $j$ assez grand, tels que pour tout $i$ avec $1 \leq i \leq t$,

$$
n_{i}=\sum_{j=1}^{\infty} \varepsilon_{i, j}\left(q^{j}-1\right) \text {. }
$$

Nécessairement, $n$ s'écrit sous la forme suivante :

$n=\sum_{j=1}^{\infty} \mu_{j}\left(q^{j}-1\right) \quad$ avec $\mu_{j} \in\{0,1, \ldots, t\}$, et $\mu_{j}=0$ pour $j$ assez grand.

Réciproquement, soit $n$ pouvant s'écrire sous cette forme. Considérons un $t$-uplet tel que $n=\sum_{i=1}^{t} n_{i}, n_{i} \geq 0$ pour tout $i$ et $\prod_{i=1}^{t} a_{0}\left(n_{i}\right) \neq 0$. Pour un tel $t$-uplet, il existe $\left(\varepsilon_{i, j}\right)$ avec $\varepsilon_{i, j}=0$ ou $1, \varepsilon_{i, j}=0$ pour tout $i$ et $j$ assez grand, tels que pour tout $i$ avec $1 \leq i \leq t$,

$$
n_{i}=\sum_{j=1}^{\infty} \varepsilon_{i, j}\left(q^{j}-1\right)
$$

$\mathrm{Or}$

$$
n=\sum_{i=1}^{t} n_{i}=\sum_{i, j} \varepsilon_{i, j}\left(q^{j}-1\right)=\sum_{j=1}^{\infty}\left(\sum_{i=1}^{t} \varepsilon_{i, j}\right)\left(q^{j}-1\right)
$$

Il résulte du lemme 1 que $\sum_{i=1}^{t} \varepsilon_{i, j}=\mu_{j}$ pour tout $j$. Par conséquent, il existe

$$
\prod_{j=1}^{\infty}\left(\begin{array}{c}
t \\
\mu_{j}
\end{array}\right)
$$

$t$-uplets de la forme cherchée. On a alors

$$
\prod_{i=1}^{t} a_{0}\left(n_{i}\right)=\prod_{i=1}^{t}(-1)^{\Sigma_{j=1}^{\infty} \varepsilon_{i, j}}=(-1)^{\Sigma_{i, j} \varepsilon_{i, j}}=(-1)^{\Sigma_{j=1}^{\infty} \mu_{j}} .
$$

Pour achever la preuve de la proposition 2, il suffit d'ajouter que l'on est en caractéristique $p$.

Nous allons déduire des propositions 1 et 2 la proposition suivante concernant les suites $f^{v}$ et $g^{v}$ : 
Proposition 3. 1. Si $f^{v}(n) \neq 0$ alors $n$ s'écrit sous la forme

$$
n=v+\sum_{j=1}^{\infty} \mu_{j}\left(q^{j}-1\right), \quad \text { avec } 0 \leq \mu_{j} \leq s .
$$

2. Si $g^{v}(n) \neq 0$ alors $n$ s'écrit sous la forme

$$
n=q+\ldots+q^{i-1}+q^{i}(v+1)+\sum_{j=1}^{\infty} \mu_{j}\left(q^{j}-1\right),
$$

avec $i \geq 1,0 \leq \mu_{j} \leq s-1$ pour $1 \leq j \leq i$, et $0 \leq \mu_{j} \leq s$ pour $j \geq i+1$.

3. Si $n=v+\sum_{j=1}^{\infty} \mu_{j}\left(q^{j}-1\right)$, avec $\mu_{j}=0$ ou $s$, pour tout $j$, et si $n$ se décompose de manière unique sous la forme (4) alors $f^{v}(n) \neq 0$.

Preuve. Preuve de 1 : Soit $n$ tel que $f^{v}(n) \neq 0$. On a

$$
f^{v}(n)=\sum_{l=0}^{n} A^{s-1}(l) a^{v}(n-l) .
$$

Soit $l$ tel que $0 \leq l \leq n$, et $A^{s-1}(l) a^{v}(n-l) \neq 0$. On a alors, d'après les propositions 1 et 2 ,

$l=\sum_{i=1}^{\infty} \delta_{i}\left(q^{i}-1\right), \quad$ avec $\delta_{i} \in\{0, \ldots, s-1\}$, et $\delta_{i}=0$ pour $i$ assez grand, $n-l=v+\sum_{j=1}^{\infty} \varepsilon_{j}\left(q^{j}-1\right), \quad$ avec $\varepsilon_{j}=0$ ou 1, et $\varepsilon_{j}=0$ pour $j$ assez grand.

Par conséquent,

$$
n=v+\sum_{i=1}^{\infty} \mu_{i}\left(q^{i}-1\right), \quad \text { avec } 0 \leq \mu_{i} \leq s .
$$

Preuve de 2 : Soit $n$ tel que $g^{v}(n) \neq 0$. On a

$$
g^{v}(n)=\sum_{l=0}^{n} A^{s-1}(l) b^{v}(n-l) .
$$

Si, pour $0 \leq l \leq n, A^{s-1}(l) b^{v}(n-l) \neq 0$, alors il existe $\left(\delta_{j}\right)_{j \in \mathbb{N}}$ avec $\delta_{j} \in$ $\{0, \ldots, s-1\}$ et $\delta_{j}=0$ pour $j$ assez grand, tels que $l=\sum_{j=1}^{\infty} \delta_{j}\left(q^{j}-1\right)$. Il existe $i \geq 1$ et $\left(\varepsilon_{j}\right)_{j \in \mathbb{N}}$ avec $\varepsilon_{j}=0$ ou 1 , et $\varepsilon_{j}=0$ pour $j$ assez grand, tels que

Par conséquent,

$$
n-l=q+\ldots+q^{i-1}+(v+1) q^{i}+\sum_{j=i+1}^{\infty} \varepsilon_{j}\left(q^{j}-1\right) .
$$

$$
n=q+\ldots+q^{i-1}+(v+1) q^{i}+\sum_{j=1}^{\infty} \mu_{j}\left(q^{j}-1\right),
$$

avec $i \geq 1,0 \leq \mu_{j} \leq s-1$ pour $1 \leq j \leq i$, et $0 \leq \mu_{j} \leq s$ pour $j \geq i+1$. 
Preuve de $3:$ Soit $n=v+\sum_{j=1}^{\infty} \mu_{j}\left(q^{j}-1\right)$, avec $\mu_{j}=0$ ou $s$, pour tout $j$, et tel que $n$ se décompose de manière unique sous la forme (4). Il existe alors un unique $l$ tel que $0 \leq l \leq n$ et tel que $A^{s-1}(l) a^{v}(n-l) \neq 0$. En effet, si $J$ est l'ensemble des indices $j$ tels que $\mu_{j} \neq 0$, on a, d'après les propositions 2 et 3.1 ,

$$
l=\sum_{j \in J}(s-1)\left(q^{j}-1\right), \quad n-l=v+\sum_{j \in J}\left(q^{j}-1\right) .
$$

On en déduit que $f^{v}(n)=A^{s-1}(l) a^{v}(n-l) \neq 0$, ce qui achève la preuve de 3 .

3. Schéma de la preuve du théorème 2. Considérons les sous-suites $\left(E\left(q^{k} n+r+(q-2)\left(q+\ldots+q^{k-1}\right)\right)\right)_{n \in \mathbb{N}}$, afin d'appliquer le théorème de Christol, Kamae, Mendès France et Rauzy, pour montrer la transcendance de $\frac{\alpha^{s}}{\Pi^{s}} \lambda(P)$. Nous supposerons $1 \leq r \leq q-2$ et nous fixerons la valeur de $r$ ultérieurement, au paragraphe 6.1.

Notons que l'on suppose $s \leq q-3$. Nous supposerons donc, jusqu'à la fin, $q \neq 2$ et 3 . La nécessité de cette limitation, pour la méthode employée, intervient dans la preuve du lemme 4 , remarque 3 , au chapitre 5 .

Nous allons étudier, à $v \geq-1$ fixé, les sous-suites $\left(f^{v}\left(q^{k} n+r+\right.\right.$ $\left.\left.(q-2)\left(q+\ldots+q^{k-1}\right)\right)\right)_{n \in \mathbb{N}}$ et $\left(g^{v}\left(q^{k} n+r+(q-2)\left(q+\ldots+q^{k-1}\right)\right)\right)_{n \in \mathbb{N}}$ et montrer les deux lemmes suivants, aux paragraphes 4 et 5 :

Lemme 3. Soit $k \geq 2$. Soient $u(k)$ et $v(k)$ les reste et quotient de la division euclidienne de $-r+q^{k}+v-(q-2)\left(q+\ldots+q^{k-1}\right)$ par s. Soit

$$
m^{v}(k)=-1+s\left(1+\ldots+q^{u(k)-1}\right)+v(k) q^{u(k)} .
$$

Pour tout $n<m^{v}(k)$, on a

$$
f^{v}\left(q^{k} n+r+(q-2)\left(q+\ldots+q^{k-1}\right)\right)=0 .
$$

Lemme 4. Soit $k \geq d+3$, où $d=\left[\frac{\ln v}{\ln q}\right]$ si $v \geq 1$, et $d=0$ sinon. Soient $x(k)$ et $y(k)$ les reste et quotient de la division euclidienne par $s$ de $-r+q^{k-d-1}-(q-3)\left(q^{k-d-2}+\ldots+q\right)-s(d+1)$. Soit

$$
n^{v}(k)=-1+s\left(1+\ldots+q^{x(k)-1}\right)+y(k) q^{x(k)} .
$$

Pour tout $n<n^{v}(k)$, on a

$$
g^{v}\left(q^{k} n+r+(q-2)\left(q+\ldots+q^{k-1}\right)\right)=0 .
$$

On a $m^{v}(k)>n^{v}(k)$ pour tout $k \geq d+3$ et $e^{v}=f^{v}+g^{v}$. Le lemme suivant résulte donc des lemmes 3 et 4 :

Lemme 5. Soit $k \geq d+3$. Pour tout $n<n^{v}(k)$, on a

$$
e^{v}\left(q^{k} n+r+(q-2)\left(q+\ldots+q^{k-1}\right)\right)=0 .
$$



tion 4

Or $E=\sum_{p_{v} \neq 0} p_{v} e^{v}$ où l'ensemble $\left\{v: p_{v} \neq 0\right\}$ est fini, d'où la proposi-

Proposition 4. Soit $n(k)=\inf \left\{n^{v}(k): v\right.$ tel que $\left.p_{v} \neq 0\right\}$. On a, pour $k$ assez grand, et tout $n<n(k)$,

$$
E\left(q^{k} n+r+(q-2)\left(q+\ldots+q^{k-1}\right)\right)=0 .
$$

Pour tout $v$, la suite $\left(n^{v}(k)\right)_{k \in \mathbb{N}}$ tend vers $\infty$. Par conséquent, la suite $(n(k))_{k \in \mathbb{N}}$ tend vers $\infty$. Les sous-suites $\left(E\left(q^{k} n+r+(q-2)\left(q+\ldots+q^{k-1}\right)\right)\right)_{n \in \mathbb{N}}$ commencent donc par une plage de 0 (non forcément maximale) dont la longueur tend vers $\infty$.

On montre, de plus, au paragraphe 6 , qu'il existe un entier $n_{0}(k)$ tel que, pour une infinité de $k, E\left(q^{k} n_{0}(k)+r+(q-2)\left(q+\ldots+q^{k-1}\right)\right) \neq 0$. Or on a le lemme suivant :

Lemme 6. Soit $\left(v_{k}\right)_{k \in \mathbb{N}}=\left(\left(v_{k}(n)\right)_{n \in \mathbb{N}}\right)_{k \in \mathbb{N}}$ une famille de sous-suites de la suite $v=(v(n))_{n \in \mathbb{N}}$ telle que :

1. Il existe un entier $m(k)$ tel que $v_{k}(n)=0$ pour tout $n<m(k)$.

2. La suite $(m(k))_{k \in \mathbb{N}}$ tend vers $\infty$.

3. Les suites $v_{k}$ sont non identiquement nulles pour une infinité de $k$.

L'ensemble $\left\{v_{k}: k \in \mathbb{N}\right\}$ est alors infini.

Il résulte de ce lemme que l'ensemble $\left\{\left(E\left(q^{k} n+r+(q-2)(q+\ldots+\right.\right.\right.$ $\left.\left.\left.\left.q^{k-1}\right)\right)\right)_{n \in \mathbb{N}}\right\}$ est infini. Du théorème de Christol, Kamae, Mendès France et Rauzy, on déduit la transcendance de $\frac{\alpha^{s}}{\Pi^{s}} \lambda(P)$ sur $\mathbf{F}_{q}(x)$ pour $q \neq 2$, 3 et $1 \leq s \leq q-3$, et, par conséquent, celle de $\lambda(P) / \Pi^{s}$, ce qui achève la preuve du théorème 2 .

Il reste à montrer le lemme 6 .

Preuve du lemme 6. D'après la condition 3, il existe un ensemble infini d'entiers $\mathcal{K}$ tel que pour tout $k$ de $\mathcal{K}$, la suite $v_{k}$ soit non nulle. Par conséquent, il existe pour tout $k$ de $\mathcal{K}$ un entier $m^{\prime}(k)$ tel que $v_{k}(n)=0$ pour tout $n<m^{\prime}(k)$, et $v_{k}\left(m^{\prime}(k)\right) \neq 0$. On a alors, d'après $1, m(k) \leq m^{\prime}(k)$.

Nous allons construire par récurrence une suite $\left(k_{j}\right)_{j \in \mathbb{N}}$ de la manière suivante :

- Soit $k_{1} \in \mathcal{K}$.

- Supposons $k_{1}, \ldots, k_{j}$ définis. Soit $k_{j+1} \in \mathcal{K}$ tel que $m\left(k_{j+1}\right)>$ $\sup \left\{m^{\prime}\left(k_{1}\right), \ldots, m^{\prime}\left(k_{j}\right)\right\}$. L'existence de $k_{j+1}$ est assurée par la condition 2.

On a alors $\sup \left\{m^{\prime}\left(k_{1}\right), \ldots, m^{\prime}\left(k_{j}\right)\right\}<m\left(k_{j+1}\right) \leq m^{\prime}\left(k_{j+1}\right)$. Par conséquent, $v_{k_{j+1}} \notin\left\{v_{k_{1}}, \ldots, v_{k_{j}}\right\}$ pour tout $j$.

On a donc construit une suite $\left(k_{j}\right)_{j \in \mathbb{N}}$ telle que $\left\{v_{k_{j}}: j \in \mathbb{N}\right\}$ est infini, ce qui achève la preuve du lemme 6 . 
4. Preuve du lemme 3. Soit $v \geq-1$ fixé. Soit $k \geq 2$. Soit $n \in \mathbb{N}$ tel que $f^{v}\left(q^{k} n+r+(q-2)\left(q+\ldots+q^{k-1}\right)\right) \neq 0$. Selon la proposition 3.1, il existe $\left(\mu_{j}\right)_{j \in \mathbb{N}}$ avec $\mu_{j} \in\{0, \ldots, s\}$ et $\mu_{j}=0$ pour $j$ assez grand, tels que

$$
q^{k} n+r+(q-2)\left(q+\ldots+q^{k-1}\right)=v+\sum_{j=1}^{\infty} \mu_{j}\left(q^{j}-1\right) .
$$

On pose $\sigma=\sum_{j=1}^{\infty} \mu_{j}$. On a

$$
q^{k} n+r+(q-2)\left(q+\ldots+q^{k-1}\right)=v+\sum_{1 \leq j \leq k-1} \mu_{j} q^{j}+\sum_{l \geq k} \mu_{l} q^{l}-\sigma .
$$

On en déduit que

avec

$$
\sigma=\lambda q^{k}+\sum_{1 \leq j \leq k-1}\left(\mu_{j}-(q-2)\right) q^{j}+v-r
$$

$$
\lambda=-n+\sum_{l \geq k} \mu_{l} q^{l-k} .
$$

Autrement dit,

$$
n=-\lambda+\sum_{l \geq k \text { et } \Sigma_{l \geq k} \mu_{l}=\mathcal{S}} \mu_{l} q^{l-k}
$$

avec

$$
\mathcal{S}=\sigma-\sum_{j<k} \mu_{j}=\lambda q^{k}+\sum_{j=1}^{k-1}\left(\mu_{j}-(q-2)\right) q^{j}-\sum_{j=1}^{k-1} \mu_{j}+v-r .
$$

Nous allons montrer que si $n$ s'écrit sous la forme (6), $n$ vérifie l'inégalité $n \geq m^{v}(k)$, avec

$$
m^{v}(k)=-1+s\left(1+\ldots+q^{u(k)-1}\right)+v(k) q^{u(k)},
$$

où $u(k)$ et $v(k)$ sont les reste et quotient de la division euclidienne par $s$ de la quantité $-r+q^{k}+v-(q-2)\left(q+\ldots+q^{k-1}\right)$. On en déduira, selon la proposition 3.1, que pour tout $n<m^{v}(k), f^{v}\left(q^{k} n+r+(q-2)\left(q+\ldots+q^{k-1}\right)\right)=0$.

Notons qu'une condition nécessaire pour que $n$ s'écrive sous la forme (6) est : $\mathcal{S} \geq 0$.

On vérifie que si $\lambda \geq 1, \mathcal{S}$ vérifie l'inégalité $\mathcal{S} \geq-r+\lambda q^{k}+v-$ $(q-2)\left(q+\ldots+q^{k-1}\right)$ et que pour $\lambda \leq 0$, on a $\mathcal{S}<0$.

Par conséquent, si $n$ s'écrit sous la forme (6), avec $\lambda=1$, on obtient $n \geq m^{v}(k)$. En revanche, pour $\lambda \geq 2$, on a, si $n$ s'écrit sous la forme (6),

$$
n \geq-\lambda+s\left(1+\ldots+q^{u^{\prime}(k)-1}\right)+v^{\prime}(k) q^{u^{\prime}(k)},
$$

où $u^{\prime}(k)$ et $v^{\prime}(k)$ sont les reste et quotient de la division euclidienne par $s$ de la quantité $-r+\lambda q^{k}+v-(q-2)\left(q+\ldots+q^{k-1}\right)$. Or on vérifie que $m^{v}(k)<-\lambda+s\left(1+\ldots+q^{u^{\prime}(k)-1}\right)+v^{\prime}(k) q^{u^{\prime}(k)}$, pour tout $\lambda \geq 2$. 
On a donc montré que pour tout $\lambda$ tel que $\mathcal{S}$ prenne des valeurs positives et donc pour tout $n$ s'écrivant sous la forme (6), on obtient $n \geq m^{v}(k)$, ce qui achève la preuve du lemme 3 .

5. Preuve du lemme 4. Soit $v \geq-1$ fixé. Soit $d=\left[\frac{\ln v}{\ln q}\right]$ si $v \geq 1$, et $d=0$ sinon. Soit $k \geq d+3$. Soit $n \in \mathbb{N}$ tel que $g^{v}\left(q^{k} n+r+(q-2)(q+\right.$ $\left.\left.\ldots+q^{k-1}\right)\right) \neq 0$. Selon la proposition 3.2, il existe $i \geq 1$ et $\left(\mu_{j}\right)_{j \in \mathbb{N}}$ avec $0 \leq \mu_{j} \leq s-1$ pour $j \leq i$, et $0 \leq \mu_{j} \leq s$ pour $j \geq i+1$, tels que $q^{k} n+r+(q-2)\left(q+\ldots+q^{k-1}\right)=q+\ldots+q^{i-1}+(v+1) q^{i}+\sum_{j=1}^{\infty} \mu_{j}\left(q^{j}-1\right)$.

On pose $\sigma=\sum_{j=1}^{\infty} \mu_{j}$.

Nous allons distinguer trois cas suivant la position de $i$ par rapport à $k$ :

Cas 1 : Si $i \geq k$, on a $\left({ }^{2}\right)$

$$
q^{k} n+r=\sum_{j=k}^{i-1} q^{j}+(v+1) q^{i}+\sum_{j=1}^{k-1}\left(\mu_{j}+1-(q-2)\right) q^{j}+\sum_{j \geq k} \mu_{j} q^{j}-\sigma .
$$

On en déduit que

$$
\sigma=\lambda q^{k}-r+\sum_{1 \leq j \leq k-1}\left(\mu_{j}+1-(q-2)\right) q^{j}-\sum_{1 \leq j \leq k-1} \mu_{j},
$$

avec

$$
\lambda=-n+\sum_{j=k}^{i-1} q^{j-k}+(v+1) q^{i-k}+\sum_{l \geq k} \mu_{l} q^{l-k} .
$$

Autrement dit,

$$
n=-\lambda+\sum_{j=k}^{i-1} q^{j-k}+(v+1) q^{i-k}+\sum_{l \geq k \text { et } \Sigma_{l \geq k} \mu_{l}=\mathcal{S}} \mu_{l} q^{l-k},
$$

avec

$$
\mathcal{S}=\lambda q^{k}-r+\sum_{1 \leq j \leq k-1}\left(\mu_{j}-(q-3)\right) q^{j}-\sum_{1 \leq j \leq k-1} \mu_{j}
$$

On montre, de même qu'au paragraphe 4 , que si $n$ s'écrit sous la forme (7), $n$ vérifie l'inégalité $n \geq n_{1}(k)$, avec $n_{1}(k)$ obtenu pour $i=k, \lambda=1$ et tel que

$$
n_{1}(k)=v+(s-1)+s\left(q+\ldots+q^{x_{1}(k)}\right)+y_{1}(k) q^{x_{1}(k)+1},
$$

où $x_{1}(k)$ et $y_{1}(k)$ sont les reste et quotient de la division euclidienne par $s$ de la quantité $q^{k}-r-(q-3)\left(q+\ldots+q^{k-1}\right)-(s-1)$. Le terme $s-1$, dans

$\left({ }^{2}\right)$ On convient, si $m<l$, de poser $\sum_{j=l}^{m} q^{j}=0$. 
l'écriture de $n_{1}(k)$, provient du fait que $\mu_{k} \leq s-1$, alors que $\mu_{j} \leq s$ pour $j \geq k+1$, quand $i=k$.

Cas 2 : Si $i=k-1$, on a

$$
q^{k} n+r=v q^{k-1}+\sum_{j=1}^{k-1}\left(\mu_{j}+1-(q-2)\right) q^{j}+\sum_{j \geq k} \mu_{j} q^{j}-\sigma .
$$

On en déduit que

$$
\sigma=-r+v q^{k-1}+\lambda q^{k}+\sum_{1 \leq j \leq k-1}\left(\mu_{j}+1-(q-2)\right) q^{j},
$$

avec $\lambda=-n+\sum_{l \geq k} \mu_{l} q^{l-k}$. Autrement dit,

$$
n=-\lambda+\sum_{l \geq k \text { et } \Sigma_{l \geq k} \mu_{l}=\mathcal{S}} \mu_{l} q^{l-k}
$$

avec

$$
\mathcal{S}=-r+v q^{k-1}+\lambda q^{k}+\sum_{1 \leq j \leq k-1}\left(\mu_{j}-(q-3)\right) q^{j}-\sum_{1 \leq j \leq k-1} \mu_{j} .
$$

Nous allons montrer que si $n$ s'écrit sous la forme (8), $n$ vérifie l'inégalité $n \geq n_{2}(k)$, avec

$$
n_{2}(k)=s\left(1+\ldots+q^{x_{2}(k)-1}\right)+y_{2}(k) q^{x_{2}(k)},
$$

où $x_{2}(k)$ et $y_{2}(k)$ sont les reste et quotient de la division euclidienne par $s$ de la quantité $-r+q^{k-1}-(q-3)\left(q^{k-2}+\ldots+q\right)-s$.

Écrivons $v$ en base $q: v=v_{0}+\ldots+v_{d} q^{d}$ avec $0 \leq v_{i} \leq q-1$ et $v_{d} \neq 0$.

Nous allons distinguer les cas, non plus selon la valeur de $\lambda$, comme au paragraphe 4 , mais selon la valeur de la quantité $v_{1}+\ldots+v_{d} q^{d-1}+\lambda$, en posant, pour $d=0, v_{1}+\ldots+v_{d} q^{d-1}=0$.

Notons que si $v_{1}+\ldots+v_{d} q^{d-1}+\lambda \leq-1$, on obtient, comme $\mu_{j} \leq s-1 \leq$ $q-4$ pour $1 \leq j \leq k-1$,

$$
\mathcal{S} \leq-r-q^{k}+v_{0} q^{k-1}-\left(q^{k-1}+\ldots+q\right)<0 .
$$

Nous allons donc supposer que $v_{1}+\ldots+v_{d} q^{d-1}+\lambda \geq 0$.

- Supposons que $v_{1}+\ldots+v_{d} q^{d-1}+\lambda \geq 1$. On a alors

$$
\mathcal{S} \geq-r+\left(v_{1}+\ldots+v_{d} q^{d-1}+\lambda\right) q^{k}+v_{0} q^{k-1}-(q-3)\left(q+\ldots+q^{k-1}\right) .
$$

Par conséquent, si $n$ s'écrit sous la forme (8) avec $v_{1}+\ldots+v_{d} q^{d-1}+\lambda \geq 1$, on obtient

$$
n \geq-\lambda+s\left(1+\ldots+q^{x_{2}^{\prime}(k)-1}\right)+y_{2}^{\prime}(k) q^{x_{2}^{\prime}(k)},
$$

où $x_{2}^{\prime}(k)$ et $y_{2}^{\prime}(k)$ sont les reste et quotient de la division euclidienne par $s$ de la quantité $-r+\left(v_{1}+\ldots+v_{d} q^{d-1}+\lambda\right) q^{k}+v_{0} q^{k-1}-(q-3)\left(q+\ldots+q^{k-1}\right)$. 
Or on vérifie que

$$
n_{2}(k)<-\lambda+s\left(1+\ldots+q^{x_{2}^{\prime}(k)-1}\right)+y_{2}^{\prime}(k) q^{x_{2}^{\prime}(k)} .
$$

- Supposons donc $v_{1}+\ldots+v_{d} q^{d-1}+\lambda=0$ et $\mathcal{S} \geq 0$. On a alors

$$
\mathcal{S}=-r+v_{0} q^{k-1}+\sum_{1 \leq j \leq k-1}\left(\mu_{j}-(q-3)\right) q^{j}-\sum_{1 \leq j \leq k-1} \mu_{j} .
$$

On a nécessairement $v_{0}+\mu_{k-1}-(q-3) \geq 1$, sinon $\mathcal{S}<0$. Par conséquent, si l'on suppose $v_{0}+\mu_{k-1}-(q-3) \geq 1$, on obtient, comme $\mu_{k-1} \leq s$,

$$
\mathcal{S} \geq-r+q^{k-1}-(q-3)\left(q+\ldots+q^{k-2}\right)-s .
$$

La condition $v_{1}+\ldots+v_{d} q^{d-1}+\lambda=0$ implique, de plus, que $\lambda \leq 0$. Par conséquent, si $n$ s'écrit sous la forme (8), avec $v_{1}+\ldots+v_{d} q^{d-1}+\lambda=0, n$ vérifie $n \geq n_{2}(k)$.

Nous avons donc montré, dans ces deux cas, l'inégalité $n \geq n_{2}(k)$, pour $n$ vérifiant (8).

Cas $3:$ Si $1 \leq i \leq k-2$, on a

$q^{k} n+r=q+\ldots+q^{i-1}+(v+1) q^{i}+\sum_{1 \leq j \leq k-1}\left(\mu_{j}-(q-2)\right) q^{j}+\sum_{l=k}^{\infty} \mu_{l} q^{l}-\sigma$.

On en déduit que

$$
\sigma=-r+\lambda q^{k}+q+\ldots+q^{i-1}+(v+1) q^{i}+\sum_{1 \leq j \leq k-1}\left(\mu_{j}-(q-2)\right) q^{j},
$$

avec $\lambda=-n+\sum_{l \geq k} \mu_{l} q^{l-k}$. Autrement dit,

$$
n=-\lambda+\sum_{l \geq k \text { et } \Sigma_{l \geq k} \mu_{l}=\mathcal{S}} \mu_{l} q^{l-k},
$$

avec

$$
\begin{aligned}
\mathcal{S} & =\sigma-\sum_{j<k} \mu_{j} \\
& =-r+\lambda q^{k}+q+\ldots+q^{i}+v q^{i}+\sum_{1 \leq j \leq k-1}\left(\mu_{j}-(q-2)\right) q^{j}-\sum_{1 \leq j \leq k-1} \mu_{j} .
\end{aligned}
$$

Nous allons montrer que si $n$ s'écrit sous la forme (9), $n$ vérifie l'inégalité $n \geq n_{3}(k)$, avec

$$
n_{3}(k)=-1+s\left(1+\ldots+q^{x_{3}(k)-1}\right)+y_{3}(k) q^{x_{3}(k)},
$$

où $x_{3}(k)$ et $y_{3}(k)$ sont les reste et quotient de la division euclidienne par $s$ de la quantité

$$
\begin{cases}-r+q^{k}-(q-2)\left(q^{k-1}+\ldots+q^{2}\right)+(v-(q-3)) q & \text { si } d=0 \\ -r+q^{k-d-1}-(q-3)\left(q^{k-d-2}+\ldots+q\right)-s(d+1) & \text { si } d \neq 0\end{cases}
$$


Nous allons raisonner, ici encore, selon les valeurs prises par $\lambda$.

- Supposons $\lambda \geq 1$. On a alors

$$
\mathcal{S} \geq-r+\lambda q^{k}-(q-2)\left(q^{k-1}+\ldots+q^{2}\right)+(v+1-(q-2)) q .
$$

Par conséquent, si $n$ s'écrit sous la forme (9) avec $\lambda \geq 1$, on obtient

$$
n \geq-\lambda+s\left(1+\ldots+q^{x_{3}^{\prime}(k)-1}\right)+y_{3}^{\prime}(k) q^{x_{3}^{\prime}(k)},
$$

où $x_{3}^{\prime}(k)$ et $y_{3}^{\prime}(k)$ sont les reste et quotient de la division euclidienne par $s$ de la quantité $-r+\lambda q^{k}-(q-2)\left(q^{k-1}+\ldots+q^{2}\right)+(v-(q-3)) q$. Or on vérifie que

$$
n_{3}(k)<-\lambda+s\left(1+\ldots+q^{x_{3}^{\prime}(k)-1}\right)+y_{3}^{\prime}(k) q^{x_{3}^{\prime}(k)} .
$$

- Supposons donc $\lambda \leq 0$. Notons que si $\lambda q^{k}+v q^{i}<q^{k-1}$, on a alors $\mathcal{S}<0$. On a, en effet $\left({ }^{3}\right)$,

$$
\mathcal{S}<-r-\left(q+\ldots+q^{k-1}\right)+\lambda q^{k}+v q^{i}<0 .
$$

Or pour $d=0$ et $\lambda \leq 0$, on a toujours $\lambda q^{k}+v q^{i}<q^{k-1}$, car $i \leq k-2$. Par conséquent, si $d=0$ et si $\mathcal{S} \geq 0$, on ne peut pas avoir $\lambda \leq 0$.

Supposons donc que $\lambda q^{k}+v q^{i} \geq q^{k-1}$. Ceci implique que $i \geq k-d-1$, car on a supposé $\lambda \leq 0$. On a alors, soit

$$
\lambda q^{k}+(v+1) q^{i}+\sum_{j \geq i}\left(\mu_{j}-(q-2)\right) q^{j} \leq 0
$$

auquel cas on a $\mathcal{S}<0$, soit

$$
\lambda q^{k}+(v+1) q^{i}+\sum_{j \geq i}\left(\mu_{j}-(q-2)\right) q^{j} \geq q^{i} .
$$

On obtient, dans ce dernier cas,

$$
\mathcal{S} \geq-r+q^{i}-(q-3)\left(q^{i-1}+\ldots+q\right)-\sum_{i \leq j \leq k-1} \mu_{j} .
$$

Or $i \geq k-d-1$ et

$$
\sum_{i \leq j \leq k-1} \mu_{j} \leq s(k-1-i)+(s-1) \leq s(d+1) .
$$

Par conséquent,

$$
\mathcal{S} \geq-r+q^{k-d-1}-(q-3)\left(q^{k-d-2}+\ldots+q\right)-s(d+1) .
$$

On a, de plus, supposé $\lambda \leq 0$. Par conséquent, si $n$ s'écrit sous la forme (9), avec $\lambda \leq 0$, on obtient $n \geq n_{3}(k)$.

Nous avons donc montré, dans tous les cas, l'inégalité $n \geq n_{3}(k)$, pour $n$ vérifiant (9).

$\left({ }^{3}\right)$ Dans cette majoration, la condition $s \leq q-3$ intervient. L'inégalité ainsi obtenue, $\lambda q^{k}+v q^{i} \geq q^{k-1}$ (c'est-à-dire, $i \geq k-d-1$ ), est essentielle pour montrer que $n \geq n_{3}(k)$. 
Il reste à comparer les quantités $n_{1}(k), n_{2}(k)$ et $n_{3}(k)$. On a, si $d \geq 1$, $n_{1}(k)>n_{2}(k)>n_{3}(k)$, et si $d=0, n_{1}(k)>n_{3}(k)>n_{2}(k)$. Soit $n^{v}(k)=$ $n_{3}(k)$ si $d \geq 1$, et $n^{v}(k)=n_{2}(k)-1$ si $d=0$. On a donc montré que pour tout $n<n_{v}(k), g^{v}\left(q^{k} n+r+(q-2)\left(q+\ldots+q^{k-1}\right)\right)=0$, avec

$$
n^{v}(k)=-1+s\left(1+\ldots+q^{x(k)-1}\right)+y(k) q^{x(k)},
$$

où $x(k)$ et $y(k)$ sont les reste et quotient de la division euclidienne par $s$ de $-r+q^{k-d-1}-(q-3)\left(q^{k-d-2}+\ldots+q\right)-s(d+1)$, ce qui achève la preuve du lemme 4 .

6. Non-nullité des suites $\left(E\left(q^{k} n+r+(q-2)\left(q+\ldots+q^{k-1}\right)\right)\right)_{n \in \mathbb{N}}$. Nous allons construire un entier $n_{0}(k)$ tel que l'on ait $E\left(q^{k} n_{0}(k)+r+\right.$ $\left.(q-2)\left(q+\ldots+q^{k-1}\right)\right) \neq 0$, pour une infinité de $k$. Nous allons définir cet entier $n_{0}(k)$ au paragraphe 6.1 , puis nous allons montrer, en posant $N_{0}(k)=q^{k} n_{0}(k)+r+(q-2)\left(q+\ldots+q^{k-1}\right)$, que $F\left(N_{0}(k)\right) \neq 0$, en 6.2 , puis que $G\left(N_{0}(k)\right)=0$, en 6.3. On en déduira que $E\left(N_{0}(k)\right) \neq 0$ et donc que les sous-suites $\left(E\left(q^{k} n+r+(q-2)\left(q+\ldots+q^{k-1}\right)\right)\right)_{n \in \mathbb{N}}$ sont non nulles pour une infinité de $k$.

6.1. Construction de l'entier $n_{0}(k)$. Soit $v$ le plus petit indice $w$ tel que $p_{w} \neq 0$. Considérons $n$ tel que

$$
f^{v}\left(q^{k} n+r+(q-2)\left(q+\ldots+q^{k-1}\right)\right) \neq 0 .
$$

On a vu, au paragraphe 4, qu'un tel $n$ s'écrit sous la forme

$$
n=-\lambda+\sum_{l \geq k \text { et } \Sigma_{l \geq k} \mu_{l}=\mathcal{S}} \mu_{l} q^{l-k}, \quad \text { avec } 0 \leq \mu_{l} \leq s, \text { pour tout } l,
$$

et

$$
\mathcal{S}=\lambda q^{k}+v-r+\sum_{j=1}^{k-1}\left(\mu_{j}-(q-2)\right) q^{j}-\sum_{j=1}^{k-1} \mu_{j} .
$$

Posons $\lambda=1$ et $\mu_{l}=0$ pour $1 \leq l \leq k-1$. On a alors

$$
\mathcal{S}=\mathcal{S}_{k}=-r+q^{k}+v-(q-2)\left(q^{k-1}+\ldots+q\right) .
$$

On peut trouver $1 \leq r \leq s$ tel que pour une infinité de $k, \mathcal{S}_{k}$ soit divisible par $s$. Nous allons donc fixer ainsi la valeur de $r$ et supposer, pour la suite, $k$ à valeurs dans un ensemble infini et tel que $\mathcal{S}_{k}$ soit divisible par $s$.

On a, de plus, $\mathcal{S}_{k} \geq s$. Il existe donc un entier $M_{k}$ tel que $\mathcal{S}_{k}=s\left(M_{k}+1\right)$. On définit alors

$$
n_{0}(k)=-1+s \sum_{j=0}^{M_{k}} q^{j} .
$$


Soit $N_{0}(k)=q^{k} n_{0}(k)+r+(q-2)\left(q+\ldots+q^{k-1}\right)$. On pose $N_{k}=M_{k}+k$. On a alors

$$
N_{0}(k)=v+s \sum_{j=k}^{N_{k}}\left(q^{j}-1\right) .
$$

6.2. Preuve de la non-nullité de $F\left(N_{0}(k)\right)$. Soit $w$ tel que $p_{w} \neq 0$. On a donc $w \geq v$. Supposons que $f^{w}\left(N_{0}(k)\right) \neq 0$. Soit $d=\left[\frac{\ln v}{\ln q}\right]$ si $v \geq 1$, et $d=0$ sinon. On définit, de même, $\Delta=\left[\frac{\ln w}{\ln q}\right]$ si $w \geq 1$, et $\Delta=0$ sinon. On a $d \leq \Delta$.

D'après la proposition 3.1 , il existe $\left(\mu_{i}\right)_{i \in \mathbb{N}}$ avec $\mu_{i} \in\{0, \ldots, s\}$, et $\mu_{i}=0$ pour $i$ assez grand, tels que

$$
N_{0}(k)=w+\sum_{i=1}^{\infty} \mu_{i}\left(q^{i}-1\right) .
$$

Or $N_{0}(k)=v+s \sum_{j=k}^{N_{k}}\left(q^{j}-1\right)$. On a donc

$$
w+\sum_{i=1}^{\infty} \mu_{i}\left(q^{i}-1\right)=v+s \sum_{j=k}^{N_{k}}\left(q^{j}-1\right) .
$$

On a alors égalité des coefficients d'indice supérieur à $k$, d'après le lemme 1 . En effet, pour $k$ assez grand, on a $k>\Delta+1 \geq d+1$ et

$$
w+\sum_{i=1}^{k-1} \mu_{i}\left(q^{i}-1\right) \leq(q-1)\left(q^{k-1}-1\right), \quad v \leq(q-1)\left(q^{k-1}-1\right) .
$$

Par conséquent, on est ramené à l'égalité suivante :

$$
v=w+\sum_{i=1}^{k-1} \mu_{i}\left(q^{i}-1\right)
$$

Or $v \leq w$. On a donc $v=w$ et $\mu_{i}=0$ pour $1 \leq i \leq k-1$.

On en déduit, d'une part, que pour $v=w$, il y a unicité de la décomposition de $N_{0}(k)$ sous la forme (10) et par conséquent, que $f^{v}\left(N_{0}(k)\right) \neq 0$, d'après la proposition 3.3. Pour $w \neq v$, on a, d'autre part, $f^{w}\left(N_{0}(k)\right)=0$. En conclusion, $F\left(N_{0}(k)\right) \neq 0$.

6.3. Preuve de la non-nullité de $G\left(N_{0}(k)\right)$. Soit $w$ tel que $p_{w} \neq 0$. Supposons que $g^{w}\left(N_{0}(k)\right) \neq 0$. Soit $\Delta=\left[\frac{\ln w}{\ln q}\right]$ si $w \geq 1$, et $\Delta=0$ sinon. On a, d'après la proposition 3.2 ,

$$
N_{0}(k)=q+\ldots+q^{i-1}+(w+1) q^{i}+\sum_{j=1}^{\infty} \mu_{j}\left(q^{j}-1\right),
$$

avec $i \geq 1,0 \leq \mu_{j} \leq s-1$ pour $1 \leq j \leq i$, et $0 \leq \mu_{j} \leq s$ pour $j \geq i+1$. 
Considérons une telle écriture. On a alors l'égalité suivante :

$$
q+\ldots+q^{i-1}+(w+1) q^{i}+\sum_{j=1}^{\infty} \mu_{j}\left(q^{j}-1\right)=v+s \sum_{j=k}^{N_{k}}\left(q^{j}-1\right) .
$$

On a vu, au paragraphe 5 , cas 1 , que si $i \geq k$, alors $q+\ldots+q^{i-1}+(w+1) q^{i}+\sum_{j=1}^{\infty} \mu_{j}\left(q^{j}-1\right) \geq q^{k} n_{1}(k)+r+(q-2)\left(q+\ldots+q^{k-1}\right)$, avec

$$
n_{1}(k)=v+(s-1)+s\left(q+\ldots+q^{x_{1}(k)}\right)+y_{1}(k) q^{x_{1}(k)+1},
$$

où $x_{1}(k)$ et $y_{1}(k)$ sont les reste et quotient de la division euclidienne par $s$ de la quantité $q^{k}-r-(q-3)\left(q+\ldots+q^{k-1}\right)-(s-1)$. Or, pour $k$ assez grand, on a :

$$
q^{k}-r-(q-2)\left(q+\ldots+q^{k-1}\right)+v<q^{k}-r-(q-3)\left(q+\ldots+q^{k-1}\right),
$$

c'est-à-dire, $n_{0}(k)<n_{1}(k)$. On ne peut donc pas avoir $N_{0}(k) \geq q^{k} n_{1}(k)+$ $r+(q-2)\left(q+\ldots+q^{k-1}\right)$, ni, a fortiori, $i \geq k$.

Nous allons distinguer deux cas selon les valeurs prises par $i$, avec $i \leq$ $k-1$, et montrer qu'ils aboutissent tous deux à une contradiction.

- Supposons $i+\Delta+1 \leq k-1$. On a donc

$$
q+\ldots+q^{i-1}+(w+1) q^{i}+\sum_{j=1}^{k-1} \mu_{j}\left(q^{j}-1\right) \leq(q-1)\left(q^{k-1}-1\right) .
$$

D'après le lemme 1 , on est alors ramené à l'égalité suivante :

$$
v=q+\ldots+q^{i-1}+(w+1) q^{i}+\sum_{j=1}^{k-1} \mu_{j}\left(q^{j}-1\right),
$$

ce qui est impossible car $v \leq w$.

- Supposons donc $k-\Delta-1 \leq i \leq k-1$. On a, pour $k$ assez grand, $i+\Delta+1 \leq N_{k}$. On est, d'après le lemme 1 , ramené à l'égalité entre les deux expressions suivantes :

$$
v+s\left(q^{k}-1\right)+\ldots+s\left(q^{i+\Delta+1}-1\right)
$$

et

$$
q+\ldots+q^{i-1}+(w+1) q^{i}+\sum_{j=1}^{i+\Delta+1} \mu_{j}\left(q^{j}-1\right) .
$$

On a, pour $i$ assez grand, donc pour $k$ assez grand, $q^{i-2}-(s(i+\Delta+2-k)) \geq 0$ et $q^{i-2}-\sum_{j=i}^{i+\Delta+1} \mu_{j} \geq 0$. En ajoutant $q^{i-2}$ aux deux membres, on obtient alors une nouvelle égalité entre les deux expressions suivantes :

$$
v+q^{i-2}-(s(i+\Delta+2-k))+s q^{k}+\ldots+s q^{i+\Delta+1}
$$


et

$$
\begin{aligned}
q+\ldots+q^{i-1} & +(w+1) q^{i} \\
& +\sum_{j=1}^{i-1} \mu_{j}\left(q^{j}-1\right)+\left(q^{i-2}-\sum_{j=i}^{i+\Delta+1} \mu_{j}\right)+\sum_{j=i}^{i+\Delta+1} \mu_{j} q^{j} .
\end{aligned}
$$

On a, de même, pour $k$ assez grand,

$$
\begin{gathered}
v+q^{i-2}-(s(i+\Delta+2-k))<q^{i} \\
q+\ldots+q^{i-1}+\sum_{j=1}^{i-1} \mu_{j}\left(q^{j}-1\right)+\left(q^{i-2}-\sum_{j=i}^{i+\Delta+1} \mu_{j}\right)<q^{i} .
\end{gathered}
$$

Effectuons alors la division euclidienne de (12) et de (13) par $q^{i}$. On a, d'après l'unicité du reste,

$v+q^{i-2}-(s(i+\Delta+2-k))=q+\ldots+q^{i-1}+\sum_{j=1}^{i-1} \mu_{j}\left(q^{j}-1\right)+\left(q^{i-2}-\sum_{j=i}^{i+\Delta+1} \mu_{j}\right)$.

Or, pour $k$ assez grand,

$$
v+q^{i-2}-(s(i+\Delta+2-k))<q^{i-1}
$$

et

$$
q+\ldots+q^{i-1}+\sum_{j=1}^{i-1} \mu_{j}\left(q^{j}-1\right)+\left(q^{i-2}-\sum_{j=i}^{i+\Delta+1} \mu_{j}\right) \geq q^{i-1} .
$$

On obtient ici encore une contradiction.

On ne peut donc pas avoir de décomposition de $N_{0}(k)$ sous la forme (11) et par conséquent, $G\left(N_{0}(k)\right)=0$.

Note (ajoutée en octobre 93). Laurent Denis m’a indiqué qu'il sait généraliser ce résultat en le déduisant du théorème de Gelfond-Schneider : il obtient ainsi la transcendance de $\lambda(P) / \pi^{r}$ pour $q \neq 2$, où $P$ est une série algébrique de degré inférieur à $q /(q-1)$ et $r$ un nombre rationnel (voir [13]).

\section{Bibliographie}

[1] J.-P. Allouche, Sur la transcendance de la série formelle $\Pi$, Sém. Théorie des Nombres de Bordeaux 2 (1990), 103-117.

[2] V. Berthé, De nouvelles preuves "automatiques" de transcendance pour la fonction zêta de Carlitz, Astérisque 209 (1992), 159-168.

[3] - Fonction zêta de Carlitz et automates, Sém. Théorie des Nombres de Bordeaux 5 (1993), 53-77.

[4] L. Carlitz, On certain functions connected with polynomials in a Galois field, Duke Math. J. 1 (1935), 137-168. 
[5] H. Chérif, Mesure d'irrationalité de valeurs de la fonction zêta de Carlitz sur $\mathbf{F}_{q}[T]$, C. R. Acad. Sci. Paris Sér. I 310 (1990), 23-26.

[6] H. Chérif and B. de Mathan, Irrationality measures of Carlitz zeta values in positive characteristic, J. Number Theory, à paraître.

[7] —, -, Mesure d'irrationalité de la valeur en 1 de la fonction zêta de Carlitz, relative $\grave{a} \mathbf{F}_{2}(T)$, C. R. Acad. Sci. Paris Sér. I 305 (1987), 761-763.

[8] G. Christol, T. Kamae, M. Mendès France et G. Rauzy, Suites algébriques, automates et substitutions, Bull. Soc. Math. France 108 (1980), 401-419.

[9] G. Dammame, Irrationalité de $\zeta(s)$ dans le corps des séries formelles $\mathbf{F}_{q}((1 / t))$, C. R. Math. Rep. Acad. Sci. Canada 9 (1987), 207-212.

[10] - , Transcendance de la fonction zêta de Carlitz par la méthode de Wade, Thèse, Caen, 1990.

[11] G. Dammame and Y. Hellegouarch, Transcendance of the values of the Carlitz zeta function by Wade's method, J. Number Theory 39 (1991), 257-278.

[12] - - - Propriétés de transcendance des valeurs de la fonction zêta de Carlitz, C. R. Acad. Sci. Paris Sér. I 307 (1988), 635-637.

[13] L. Denis, Méthodes fonctionnelles pour la transcendance en caractéristique finie, prépublication.

[14] V. G. Drinfeld, Elliptic modules, Mat. Sb. 94 (136) (1974), 594-627 (en russe); trad. angl. : Math. USSR-Sb. 23 (1974), 561-592.

[15] E. U. Gekeler, Drinfeld Modular Curves, Lecture Notes in Math. 1231, Springer, 1986.

[16] F. Recher, Propriétés de transcendance de séries formelles provenant de l'exponentielle de Carlitz, C. R. Acad. Sci. Paris Sér. I 315 (1992), 245-250.

[17] D. S. Thakur, Gauss functions and Gauss sums for function fields and periods of Drinfeld modules, Ph.D. thesis, Harvard, 1987.

[18] —, Number fields and function fields, dans : N. De Grande-De Kimpe and L. Van Hamme (eds.), Proc. Conf. on $p$-adic Analysis, Hengelhoef, 1986, Vrije Universiteit, Brussels, 149-157.

[19] L. J. Wade, Certain quantities transcendental over $G F\left(p^{n}, x\right)$, Duke Math. J. 8 (1941), 701-720.

[20] - Transcendence properties of the Carlitz $\psi$-function, ibid. 13 (1946), 79-85.

[21] M. Waldschmidt, Transcendence problems connected with Drinfeld modules, İstanbul Üniv. Fen Fak. Mecm. Ser. A 49 (1990), 57-75.

[22] J. Yu, Transcendence and special zeta values in characteristic p, Ann. of Math. 134 (1991), 1-23.

[23] - , Transcendental numbers arising from Drinfeld modules, Mathematika 30 (1983), 61-66.

[24] - , Transcendental theory over function fields, Duke Math. J. 52 (1985), 517-527.

LABORATOIRE DE MATHÉMATIQUES DISCRÈTES

LUMINY CASE 930

13288 MARSEILLE CEDEX 9, FRANCE 